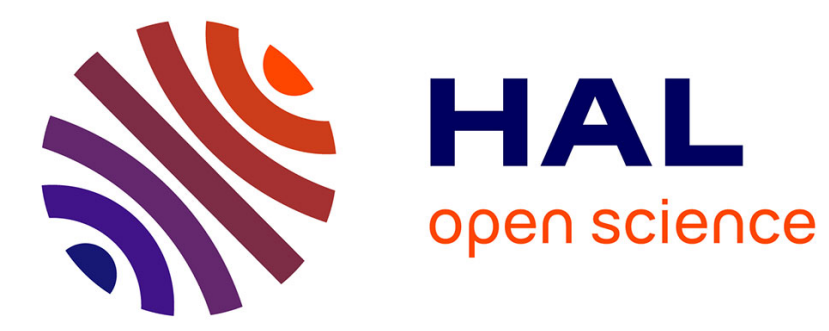

\title{
Phonon-assisted transitions of bound excitons in diamond: Analysis by mirror symmetry
}

Yoshiki Kubo, Mitsuyoshi Takahata, Solange Temgoua, Riadh Issaoui, Julien Barjon, Nobuko Naka

\section{- To cite this version:}

Yoshiki Kubo, Mitsuyoshi Takahata, Solange Temgoua, Riadh Issaoui, Julien Barjon, et al.. Phononassisted transitions of bound excitons in diamond: Analysis by mirror symmetry. Physical Review B, 2020, 101 (20), 10.1103/PhysRevB.101.205204 . hal-02976785

\section{HAL Id: hal-02976785 \\ https://hal.science/hal-02976785}

Submitted on 24 Nov 2020

HAL is a multi-disciplinary open access archive for the deposit and dissemination of scientific research documents, whether they are published or not. The documents may come from teaching and research institutions in France or abroad, or from public or private research centers.
L'archive ouverte pluridisciplinaire HAL, est destinée au dépôt et à la diffusion de documents scientifiques de niveau recherche, publiés ou non, émanant des établissements d'enseignement et de recherche français ou étrangers, des laboratoires publics ou privés. 


\title{
Phonon-assisted transitions of bound excitons in diamond: Analysis by mirror symmetry
}

\author{
Yoshiki Kubo, ${ }^{1}$ Mitsuyoshi Takahata, ${ }^{1}$ Solange Temgoua, ${ }^{2}$ Riadh Issaoui $\odot,{ }^{3}$ Julien Barjon, ${ }^{2,}{ }^{*}$ and Nobuko Naka $\oplus^{1, \dagger}$ \\ ${ }^{1}$ Department of Physics, Kyoto University, Kitashirakawa-Oiwake-cho, Sakyo-ku, Kyoto 606-8502, Japan \\ ${ }^{2}$ Université Paris-Saclay, UVSQ, CNRS, GEMaC, 78000 Versailles, France \\ ${ }^{3}$ LSPM-CNRS, Université Sorbonne Paris Nord, 99 Avenue Jean-Baptiste Clément, 93430 Villetaneuse, France
}

(Received 22 October 2019; revised manuscript received 7 April 2020; accepted 13 April 2020; published 11 May 2020)

\begin{abstract}
This study aims at a quantitative understanding of the optical spectra taken from bound excitons weakly coupled to phonons in indirect semiconductors, which had been missing for decades. Insights on the properties of excitons bound at neutral acceptor impurities are obtained by analyzing the spectra reported for diamond with appropriate boron doping [Y. Kubo et al., Appl. Phys. Lett. 114, 132104 (2019)]. We focus on the mirror symmetry holding between phonon-assisted absorption and luminescence simultaneously regarding energies, linewidths, and intensities. New analytic expressions are proposed to reproduce the spectra of phonon-assisted recombination lines. The detailed analysis reveals the contribution from excited bound-exciton states whose origin is discussed and modeled. Taking them into account improves simulation of the bound-exciton optical spectra in indirect band-gap semiconductors up to a quantitative level.
\end{abstract}

DOI: 10.1103/PhysRevB.101.205204

\section{INTRODUCTION}

Some types of defects in crystalline materials act as localized centers and lead to the characteristic optical absorption and emission. The most widely studied examples are paramagnetic impurities in ionic crystals (e.g., rubies - chromium impurities in sapphire) [1] and vacancy complexes (color centers) in alkali halides [2]. The electronic excitations of these localized centers, such as Frenkel excitons, strongly couple with phonons in the host material to form vibronic systems. These systems typically comprise harmonic oscillator potentials with a series of equally spaced energy levels, both for the initial and final electronic states of the optical transitions. In atomic transitions, the emission and absorption frequencies coincide. Contrary to this, the emission in a vibronic system generally occurs at a lower energy than the absorption, where the difference is referred to as the Stokes shift. The spectra are characterized by the phonon wing consisting of a series of broad lines equally separated by the energy of local vibrational modes. As can be understood by the configuration coordinate diagram, the absorption and emission spectra appear in mirror symmetry about the nophonon (NP) line position [3].

The case with a weaker (medium and weak) electronphonon coupling is less obvious. The weak coupling regime is realized with shallow centers in nonpolar materials holding inversion symmetry, in which the two most efficient phonon coupling mechanisms-Fröhlich and piezoelectric interactions-are missing [3]. A representative example of the weak coupling (Huang-Rhys factor $S \leqslant 1$ ) is the excitons bound to impurity centers in indirect band-gap

\footnotetext{
*julien.barjon@uvsq.fr

†naka@scphys.kyoto-u.ac.jp
}

semiconductors, which were observed with the shallow dopants of silicon [4] already in the 1960s. Although free (delocalized) indirect excitons require phonon assistance for the optical transitions, the introduction of the impurity centers lowers translational symmetry of the crystal, allowing for the $\mathrm{NP}$ lines of the bound excitons to appear together with the phonon-assisted optical transition lines [5,6].

The phonon-assisted transitions are separated from the NP line by the energies of bulk phonons, and referred to as phonon replicas instead of the phonon wings in the strong coupling case. Absorption spectra of bound excitons in silicon have been available only from samples with much higher doping concentrations than for emission, e.g., 100 times higher $[7,8]$. At such high doping levels, the absorption lines suffer concentration broadening due to the neighboring impurities [7,9]. Therefore, the mirror symmetry between emission and absorption spectra of bound excitons in the weak coupling regime could not be investigated in any detail up to now $[8,10,11]$.

In this article, we present our analysis of the phonon replicas of excitons bound to neutral impurity centers derived from boron atoms doped in diamond [12]. This indirect bandgap material possesses similar crystal and band structures to silicon, and thus nonpolar and centrosymmetric features leading to the weak exciton-phonon coupling. The analysis was possible owing to the measurements of the bound-exciton absorption spectrum, which had been missing till very recently for diamond [13]. In the following sections, absorption and emission (luminescence) spectra measured on the same high-quality diamond sample chosen for an appropriate doping concentration are analyzed by using the spectral mirror symmetry. Well-isolated phonon replicas were observed in an unprecedented condition that intrinsic phonon broadening surpasses concentration broadening (Sec. II). Our spectral analysis reveals that the phonon replicas in diamond exhibit 


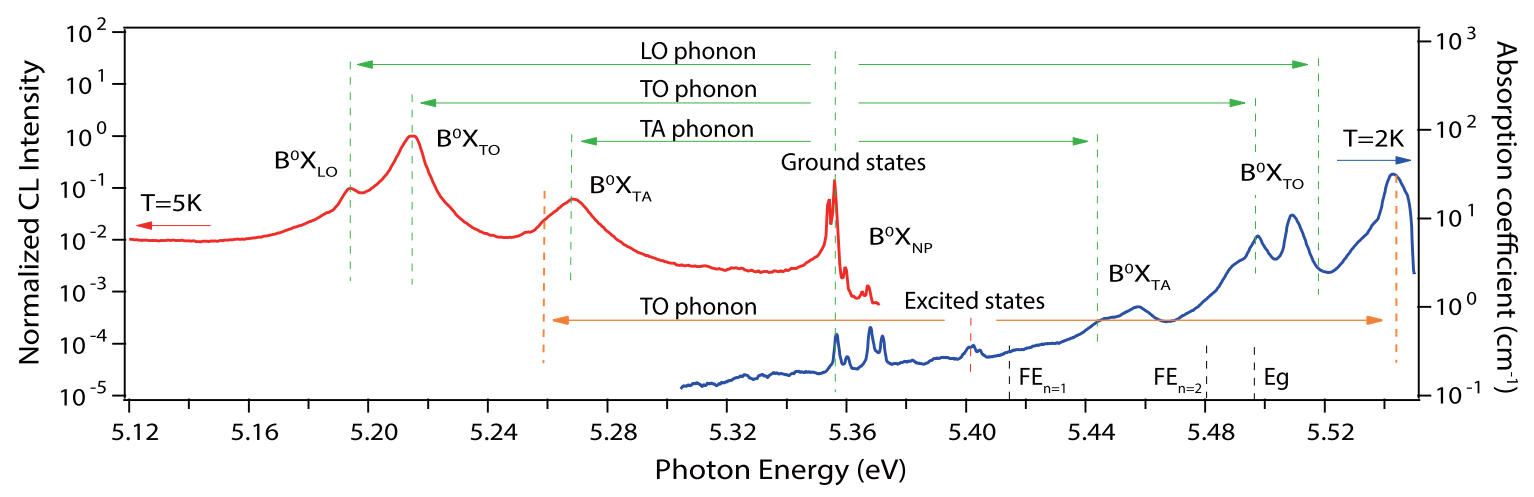

FIG. 1. Overview of cathodoluminescence and absorption spectra of bound excitons in boron-doped diamond at low temperatures (reproduced from Ref. [13]). The dashed lines indicate the positions of NP and phonon replica lines, expected positions of the free exciton (FE) states [18], and the band gap $E_{g}$. Note that the LO phonon replica is only seen in luminescence since its absorption counterpart is masked by the TO absorption tail.

clear deviation from the first approximation approach used in silicon [6] (Sec. III). By comparison of the data with calculations including the phonon dispersions and bound exciton wave functions, the excited states of the bound exciton at the impurity center could be revealed and their origin is discussed (Sec. IV).

\section{EXPERIMENTS}

An absorption spectrum of boron-doped diamond measured at $2 \mathrm{~K}$ is shown in Fig. 1. The figure is reproduced from Ref. [13] with the change from linear to logarithmic scales. A 251- $\mu \mathrm{m}$-thick diamond film with the boron concentration of $1.8 \times 10^{18} \mathrm{~B}$ atoms $\mathrm{cm}^{-3}$, grown by the plasma-assisted chemical-vapor-deposition method [14], was used. Other experimental details are described elsewhere [13]. To highlight the bound exciton contributions, absorption due to free excitons has been subtracted by using a spectrum of an intrinsic diamond. A cathodoluminescence spectrum obtained at $5 \mathrm{~K}$ is also shown for comparison. As reported in our previous study [13], the sharp peaks around $5.36 \mathrm{eV}$ are NP lines of the bound exciton. The first-order phonon replica lines, corresponding to the emission of different types of phonons, are indicated by the arrows. The subscript to $B^{0} X$ denotes NP or the phonon modes: longitudinal optical (LO), transverse optical (TO), and transverse acoustic (TA). Near $5.40 \mathrm{eV}$ at an energy about 40 $\mathrm{meV}$ higher than the ground state NP lines, we additionally found a group of narrow lines. These lines are attributed to excited states of the bound exciton as will be discussed in Sec. IV.

\section{RESULTS}

Boron atoms introduced in a diamond crystal lattice act as acceptor impurities, and serve as optical centers by capturing free excitons. A neutral boron-bound exciton $\left(B^{0} X\right)$ is regarded as a complex of four particles, i.e., one electron and two holes bound to a negative ionic potential centered at zero around the acceptor impurity fixed in the crystal lattice. The bound exciton in the initial state recombines by emitting a photon leaving one of the holes on the acceptor potential, with the final state being a neutral acceptor state $B^{0}$. In absorption, the initial and final states of the optical transition are reversed.

At low temperature, the transition lines reveal the finalstate splitting since only the lowest-energy level of the initial states is significantly occupied. Namely, the NP structures in luminescence and absorption reflect the fine structure of the acceptor state and that of the bound exciton, respectively.

The full lines in Figs. 2(a) and 2(b) show the luminescence and absorption spectra plotted on a linear scale. As a first step, we tried to reproduce these spectra by using a simple empirical function.

\section{A. Luminescence}

The NP lines in the luminescence spectrum of Fig. 2(a) consist mainly of a doublet structure associated with the final-state splitting due to spin-orbit interaction $(2.07 \mathrm{meV})$ in the acceptor state [15]. The NP luminescence lines are well reproduced by a double Gaussian line as shown by the full green line in Fig. 2(a) on a horizontally expanded scale. A larger linewidth of $1.1 \mathrm{meV}$ was found for the low-energy peak than for the high-energy peak of $0.9 \mathrm{meV}$ width, which was limited by the spectral resolution. The higher-energy components can be distinguished in the logarithmic plot in Fig. 1 and are consistent with previous observations [16,17]. Further, the luminescence spectra recorded at higher temperatures are presented and analyzed in the Appendix.

Compared to NP lines, phonon replica lines of bound excitons generally present much larger linewidths due to phonon broadening. When a Gaussian broadening is assumed as initially done by Thewalt et al. in silicon [6], there are deviations at the top and tails [see the inset of Fig. 2(a)]. We found out that the lineshape of a phonon replica is better reproduced by a Laplacian broadening,

$$
L(E)=\frac{A_{v}}{2 W_{v}} \exp \left(-\frac{\left|E-E_{0}\right|}{W_{v}}\right),
$$

rather than by the Gaussian broadening. Here $E$ is the photon energy, $E_{0}$ is the center position of the peak, and $2 W_{\nu} \ln 2$ is the full width at half maximum (FWHM) of the broadening function depending on the phonon mode $v$. We should note 
(a)

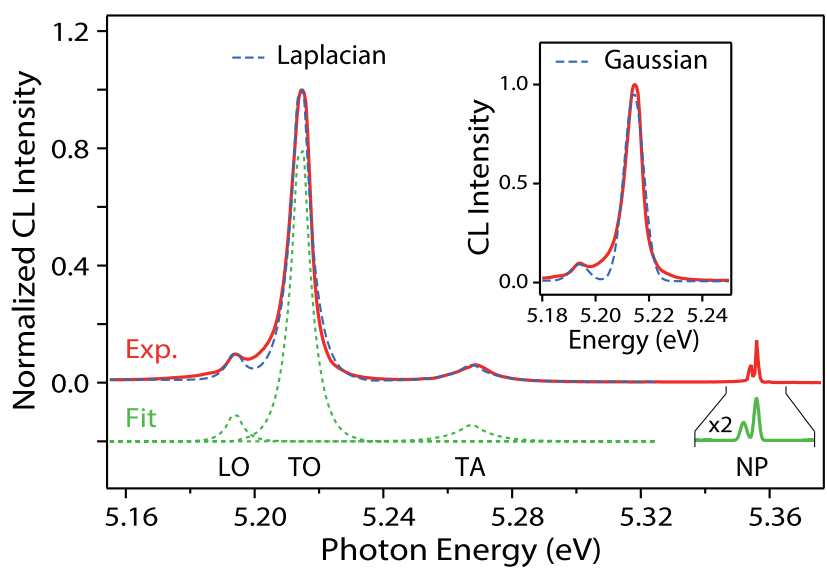

(b)

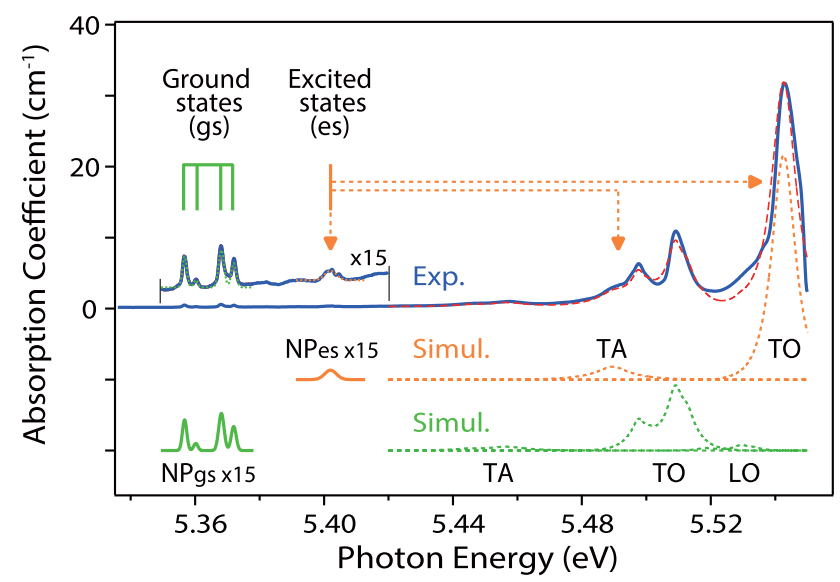

FIG. 2. Simulations of optical spectra for the boron-bound exciton in diamond: (a) Luminescence spectrum measured at $5 \mathrm{~K}$ (full red line) in comparison with the fitted curves based on a Laplacian broadening (dashed line). The lower spectrum evidences the contribution of each phonon, separately (dotted lines). The simulated NP lines are enlarged horizontally. The inset presents the best fit obtained by assuming a Gaussian broadening for TO and LO replicas. (b) Absorption spectrum measured at $2 \mathrm{~K}$ (full blue line) in comparison with simulated curves (dashed line) obtained by mirror symmetry of luminescence (details in the text). The bottom and middle spectra distinguish the ground state (green) and excited state (orange) contributions to the absorption spectrum. The NP lines are shown on an expanded vertical scale.

that the choice of this new function is empirical and might appear not totally physical due to the discontinuity of its derivative at $E=E_{0}$. Still, it remains a reasonable description of phonon replica lines since the sharp point at its peak is smoothed when convoluted by the Gaussian shape of NP lines. At the end, the integrated intensity of phonon replica lines is $I_{v}=I_{\mathrm{NP}} \times A_{v}$.

The dashed line in Fig. 2(a) represents a simulated luminescence spectrum. The method is the following: Each phonon replica was numerically calculated by convoluting the NP lines with a Laplace function [Eq. (1)] having its center energy reduced with respect to NP lines by the amount of the corresponding phonon energy $(88,141$, and $162 \mathrm{meV}$ for TA, TO, and LO phonons $[17,18])$. The linewidths $W_{\nu}$ and spectral areas $A_{v}$ are adjustable parameters. A best fit of the experimental data is obtained for the parameter set given by the relations

$$
\begin{aligned}
& A_{\mathrm{LO}}: A_{\mathrm{TO}}: A_{\mathrm{TA}}=0.08: 1: 0.09, \\
& W_{\mathrm{LO}}: W_{\mathrm{TO}}: W_{\mathrm{TA}}=0.8: 1: 1.8,
\end{aligned}
$$

with $A_{\mathrm{TO}}=36$ and 65 , respectively, for the low- and highenergy NP lines, and $W_{\mathrm{TO}}=3.7 \mathrm{meV}(\mathrm{FWHM}=5.1 \mathrm{meV})$ for both lines.

The agreement with the measured spectrum is quite satisfactory. Therefore, the proposed empirical function (Laplacian) provides a fair simulation of phonon broadening in the luminescence spectra of bound excitons in diamond, and might apply as well to other indirect band-gap semiconductors.

The $A_{v}$ values found for respective phonon replicas indicate the probabilities of each phonon to assist the radiative recombination of the boron-bound exciton in diamond. Their quantitative understanding requires a microscopic theory, which is beyond the scope of this paper. On the other hand, the phonon replica linewidths result from the phonon dispersion and the spread of the bound-exciton wave function in the reciprocal space, as further discussed in Sec. IV.

\section{B. Absorption}

Spectral simulations are performed in a similar manner for the absorption spectrum using Laplace functions with the linewidths $W_{\mathrm{LO}}, W_{\mathrm{TO}}, W_{\mathrm{TA}}$ and the relative coupling strengths $A_{\mathrm{LO}}: A_{\mathrm{TO}}: A_{\mathrm{TA}}$, both extracted from the luminescence fit.

Note that the procedure is not as straightforward due to a more complex structure of NP transitions in absorption. While luminescence involves only the lowest-energy states of the bound exciton, absorption results from all its energy states. In absorption, the NP lines exhibit a quadruplet splitting associated to the four-fold ground state of the boron-bound exciton in diamond [see the left upper part of Fig. 2(b)].

The NP lines observed in absorption are reproduced by the sum of four Gaussian lines in the bottom spectrum in Fig. 2(b). The TA, TO, and LO replica lines were calculated by assuming mirror symmetry to the luminescence regarding the energy positions, linewidths, and relative intensities among different phonon replicas. Note that among the quadruplet observed in absorption, only the lowest-energy line at $5.356 \mathrm{eV}$ is common to the luminescence spectrum, where it corresponds to the higher-energy line of the doublet. The phonon replica of this common NP line is described with exactly the same parameter $\left(A_{\mathrm{TO}}=65\right)$ in absorption and luminescence simulations. The phonon coupling strengths for other quadruplet lines were found to be equal except for the third line $\left(A_{\mathrm{TO}}=\right.$ 90), for which there is no possible comparison since it is not observed in luminescence.

With this approach, some features of the observed absorption spectrum are correctly described [green curves in Fig. 2(b)]. However, several peaks of the experimental absorption are not reproduced, as indicated by three arrows near 5.40, 5.49, and $5.54 \mathrm{eV}$ in Fig. 2(b). They include the dominant peak observed at $5.54 \mathrm{eV}$ with an absorption coefficient reaching $30 \mathrm{~cm}^{-1}$. The energy and linewidth of the 
three peaks are consistent with NP, TA, and TO replica lines of a bound exciton with higher energy. This reveals the major contribution from excited states of the bound exciton in the absorption spectrum [19].

In the middle spectrum of Fig. 2(b), the NP line of the excited states is approximated by a single Gaussian line, although it consists of a few overlapping narrow components. The TA and TO replicas of the NP line are calculated similarly to the ground-state ones, i.e., with a Laplacian broadening of NP lines. The best agreement of our simulations with absorption data is obtained for ground- and excited-state parameters that verify for the NP lines,

$$
I_{\mathrm{NP}}^{g s}: I_{\mathrm{NP}}^{e s}=1: 0.16 \pm 0.04,
$$

and for the phonon replicas,

$$
\begin{aligned}
& A_{v}^{g s}: A_{v}^{e s}=1: 12.3 \pm 0.3, \\
& W_{v}^{g s}: W_{v}^{e s}=1: 1,
\end{aligned}
$$

where the superscripts ( $g s$ and $e s$ ) refer to the ground and excited states, respectively. The sum of the ground- and excitedstate components is represented by the dashed line in the top spectrum of Fig. 2(b), which is almost superimposed to the experimental data. This work on diamond demonstrates that the simulations of absorption spectra can reach a quantitative level for bound excitons in indirect semiconductors.

Thanks to the mirror-symmetry approach developed in this work, the contribution of excited states in the bound exciton absorption spectrum has been evidenced. The relative absorption intensities of ground and excited states that were extracted will contribute to the discussion on their origin, as developed in Sec. IV B.

\section{DISCUSSION}

In the first part of the section, the origin of the spectral shape observed and simulated for the phonon replicas of bound excitons is discussed from the physical point of view. The discussion will be related to the luminescence results, i.e., mainly on optical transitions starting from the bound-exciton ground states. In the second part, we will come back on the excited states observed in absorption to understand their binding mechanism.

\section{A. Phonon broadening}

Here we will discuss the linewidths of the phonon replica lines for the ground states. We follow the approach presented in Refs. $[6,17]$ and assume the following. (i) The envelope function of the bound exciton states is expressed by the wave function $F(\mathbf{K})$ that is spherically symmetrical around the conduction band minimum at $\mathbf{K}=\mathbf{k}_{0}=\left(0,0, k_{0 z}\right)$. (ii) The interaction between the bound exciton and phonon does not depend on the phonon wave vector around $\mathbf{k}_{0}$, so the squared matrix element for the transition is proportional to the squared amplitude of the wave function.

Figure 3(a) shows dispersion relations of LO, TO, LA, and TA phonons along the $\Gamma-X$ axis [20]. The gradients of the phonon dispersion at $\mathbf{k}_{0}$ ( 0.76 of the $X$ point) are dominant along the $\Gamma-X$ axis [6], so that the dispersion can be expressed as $E_{p h}=E_{v}+g_{v}\left(K_{z}-k_{0 z}\right)$ with $K_{z}$ being the only variable. The energy conservation for the optical transitions is (a)

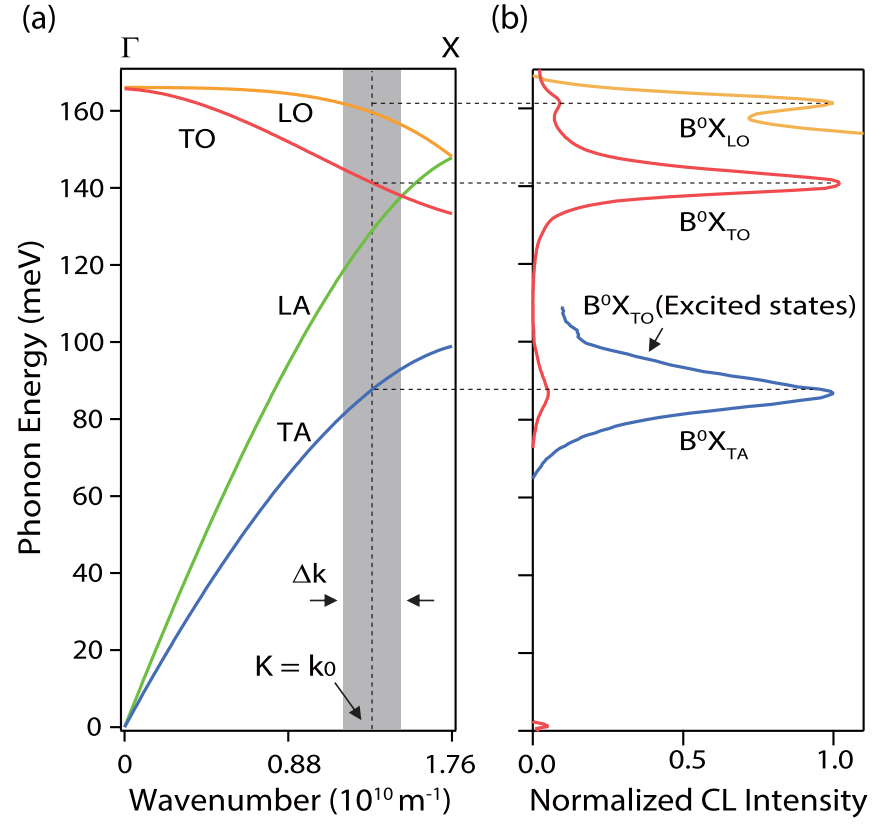

FIG. 3. (a) Phonon dispersion of diamond (polynomial fits to the data in Ref. [20]). (b) Phonon replicas of the luminescence at $5 \mathrm{~K}$ with the vertical axis representing the energy difference from the center of the NP lines. Three curves are identical data but normalized by different factors.

expressed by

$$
E^{ \pm}=E_{\mathrm{NP}} \pm E_{p h}=E_{\mathrm{NP}} \pm E_{v} \pm g_{v}\left(K_{z}-k_{0 z}\right),
$$

where $E^{ \pm}$is the energy of the photon absorbed (positive sign) or emitted (negative sign). $E_{\mathrm{NP}}=E_{g}-E_{x}-E_{\mathrm{loc}}$ is the NP line position, where $E_{g}$ is the indirect bandgap energy, $E_{x}$ the exciton binding energy, and $E_{\text {loc }}$ the localization energy of the bound exciton. Under assumptions (i) and (ii) given above, the spectral width is scaled by the gradient $\left|g_{v}\right|$ and by the width of $F(\mathbf{K})$. The gradients calculated by taking the derivatives of the phonon dispersions are

$$
\left|g_{\mathrm{LO}}\right|:\left|g_{\mathrm{TO}}\right|:\left|g_{\mathrm{TA}}\right|=0.72: 1.0: 1.6
$$

where $g_{\mathrm{TO}}=2.5 \times 10^{-12} \mathrm{eV} \mathrm{m}$. These values agree well with the ratio we obtained with Laplacian fits in Sec. III for the linewidths $W_{v}$ when considering the fitting error of approximately $10 \%$.

Figure $3(\mathrm{~b})$ is drawn to graphically highlight this numerical agreement. At the same energy scale as in Fig. 3(a), it shows the LO, TO, and TA replicas of the bound exciton luminescence, the zero energy being at the center of gravity of the two NP lines. It can be seen that the FWHMs of phonon replica lines are governed by the momentum broadening of the bound exciton $\Delta k=\mathrm{FWHM} /\left|g_{v}\right|=2 \times 10^{9} \mathrm{~m}^{-1}$ due to its localization in real space. The momentum range $\Delta k$ is shaded in Fig. 3(a). It is approximately three times that in silicon [6] and is responsible for the larger phonon broadening observed in diamond. Note that the small shoulder at the tail of the TA phonon replica is considered as the TO phonon replica of the excited states. This component is more pronounced when 
thermally activated in luminescence at higher temperatures [see the Appendix].

To summarize, the linewidths of phonon replicas appear as consequences of the intrinsic phonon properties and the spread of the bound-exciton wave function in the reciprocal space. The large group velocities of phonons in diamond have been helpful to differentiate the specific broadening of each phonon mode. Another favorable factor compared to the silicon case is a stronger localization of the bound exciton wave functions, which limits the concentration broadening to a second-order effect in this study.

\section{B. Excited states of bound excitons}

In this last part, we discuss the origin of the new lines revealed in the absorption spectrum of boron-bound excitons thanks to the mirror symmetry analysis based on luminescence data. To that end, we extend the model proposed in Ref. [21] for direct band-gap semiconductors to indirect ones and express the wave function of an acceptor-bound exciton as

$$
\Psi_{A^{0} X}=\phi_{A}\left(\mathbf{r}_{h 1}\right) \psi_{F E}\left(\mathbf{r}_{h 2}-\mathbf{r}_{e}\right) \Phi(\mathbf{R}),
$$

where $\phi_{A}\left(\mathbf{r}_{h 1}\right)$ is the hole wave function of the acceptor state and $\psi_{\mathrm{FE}}\left(\mathbf{r}_{h 2}-\mathbf{r}_{e}\right)$ describes the internal motion of the free exciton state. $\Phi(\mathbf{R})$ is the envelope state of the quasifree exciton orbiting as an entity around the neutral acceptor, with $\mathbf{R}$ being the center-of-mass coordinate of the electron and hole $[21,22]$. While the hole-hole exchange interaction is not explicitly included in this simplified equation, we treat the two holes as identical particles and take the hole wave function to be symmetric in the coordinates $\mathbf{r}_{h 1}$ and $\mathbf{r}_{h 2}$.

Two different kinds of bound-exciton excited states are known from the literature [22]: one with the $n=2$ orbital state of a free exciton weakly bound to the impurity as an entity [Fig. 4(a)], and the other with the internal $n=2$ state of the electron in a free exciton [Fig. 4(b)], where $n$ is the principal quantum number. The former state appears at a slightly higher energy than the ground state, whereas the latter state appears at a slightly lower energy than the $n=2$ free exciton state since $E_{\mathrm{loc}}<E_{x}[23]$.

If the present excited states were an internal $n=2$ state of the electron in the exciton, they should be located just below the $n=2$ free exciton state [22] labeled $\mathrm{FE}_{n=2}$ in Fig. 1. The corresponding excited state of $\psi_{\mathrm{FE}}$ is schematized in Fig. 4(b). Considering on the contrary that the spectral feature is detected experimentally at $5.40 \mathrm{eV}$, i.e., at a much lower energy than $\mathrm{FE}_{n=2}$, the present excited states are rather believed to be the $n=2$ orbital state of the quasifree exciton, $\Phi(\mathbf{R})$, as illustrated in Fig. 4(a).

We propose now to turn this model into figures, considering that the envelope function $\Phi(\mathbf{R})$ has approximate solutions in the case $E_{\text {loc }}<E_{x}[17,22]$, which tend to the hydrogen atom wave functions at large distances from the neutral acceptor. In the following, we propose to use the radial wave functions of the hydrogen atom to describe the envelope function $\Phi(\mathbf{R})$ at large distances. This function contains the radial part of the wave function only and does not include the angular part. The Fourier transform of $\Phi(\mathbf{R})$ is then described (a)

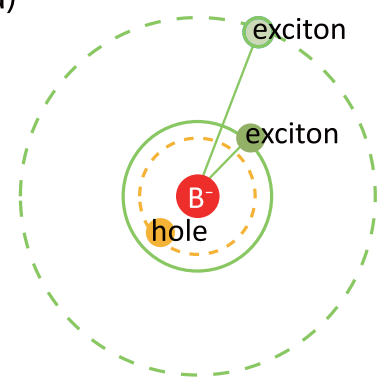

(b)

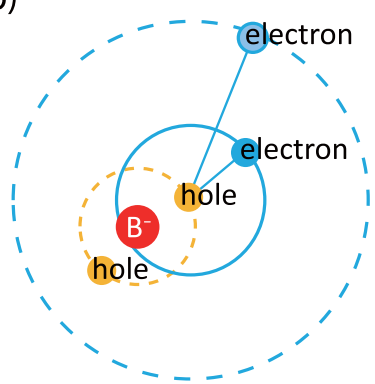

(c)

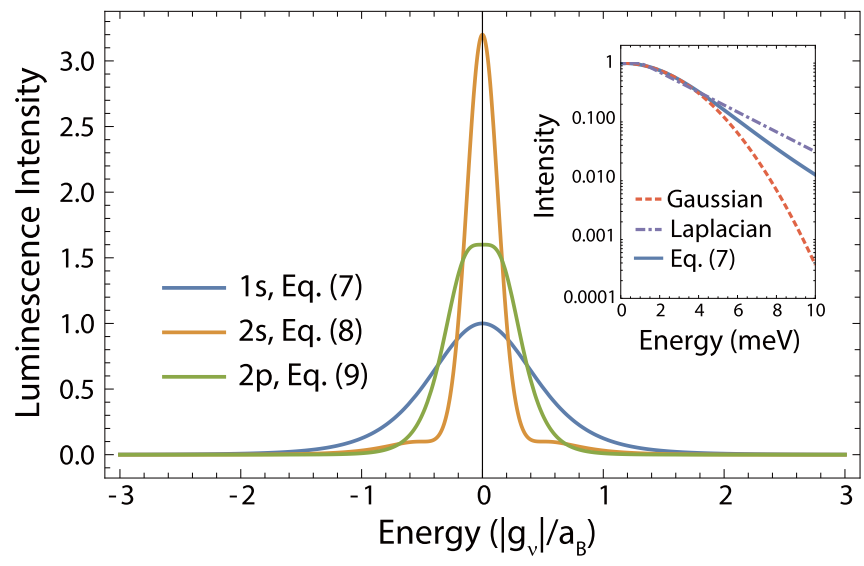

FIG. 4. (a,b) Schematics illustrating two kinds of excited states of a bound exciton. Orbitals for the ground and excited states are schematically shown by solid and dashed circles. In (a) shown is an excited envelope state of the quasifree exciton orbiting around the neutral acceptor. The acceptor hole and the hole in the quasifree exciton are assumed to take symmetric coordinates. In (b) shown is an excited state within the internal state of the quasifree exciton. (c) Spectral shapes of the phonon replica, Eqs. (7) to (9). Inset shows the $1 s$ spectral shape [Eq. (7), full line] in comparison with empirical broadenings: a Gaussian (dashed line) and a Laplacian (dashed-dotted line). All curves in the inset were calculated with an energy unit of $\left|g_{v}\right| / a_{B}=5 \mathrm{meV}$ and respectively convoluted by the NP lines.

as [24-26]

$$
F_{1 s}(\mathbf{K})=\frac{2^{5 / 2} a_{B}^{3 / 2}}{\sqrt{\pi}\left(1+a_{B}^{2}\left|\mathbf{K}-\mathbf{k}_{0}\right|^{2}\right)^{2}},
$$

for the $1 s$ state

$$
F_{2 s}(\mathbf{K})=\frac{2^{5} a_{B}^{3 / 2}\left(1-4 a_{B}^{2}\left|\mathbf{K}-\mathbf{k}_{0}\right|^{2}\right)}{\sqrt{\pi}\left(1+4 a_{B}^{2}\left|\mathbf{K}-\mathbf{k}_{0}\right|^{2}\right)^{3}},
$$

for the $2 s$ state, and

$$
F_{2 p}(\mathbf{K})=\frac{2^{7} a_{B}^{5 / 2}\left|\mathbf{K}-\mathbf{k}_{0}\right|}{\sqrt{3 \pi}\left(1+4 a_{B}^{2}\left|\mathbf{K}-\mathbf{k}_{0}\right|^{2}\right)^{3}},
$$

for the $2 p$ state, where $a_{B}$ is an effective Bohr radius for the envelope function. It then provides the mean distance between the quasifree exciton and the neutral acceptor, which is $3 a_{B} / 2$ for the $1 s$ state. We centered these functions at $\mathbf{k}_{0}$ to take account of the indirect band-gap property of diamond. The squared amplitude of $F(\mathbf{K})$ at $\mathbf{K}=0$ determines probabilities 
of the NP transitions. It is remarkable that the intensity of the $2 p$ NP line vanishes $\left|F_{2 p}(0)\right|^{2} /\left|F_{1 s}(0)\right|^{2} \approx 0$ when $a_{B}\left|\mathbf{k}_{0}\right| \gg 1$, so the NP excited states which are observed near $5.40 \mathrm{eV}$ in absorption should rather have a $2 s$ origin. The ratio of the NP line intensities between the ground state $(1 s)$ and excited state $(2 s)$ is calculated as

$$
\left|F_{1 s}(0)\right|^{2}:\left|F_{2 s}(0)\right|^{2}=1: 0.125 \text {, }
$$

in the limit of $a_{B}\left|\mathbf{k}_{0}\right| \gg 1$. Such a result is not surprising since the squared amplitude at the origin $|\Phi(0)|^{2}$ scales with $1 / n^{3}$ for $s$-envelope states. The precise value of the effective Bohr radius $a_{B}$ of the envelope function remains unknown in diamond, but it is reasonably expected in the nanometer range which is consistent with the limit case considered $\left(a_{B}=1 \mathrm{~nm}\right.$ gives $a_{B}\left|\mathbf{k}_{0}\right|=13.2$ ).

The model proposed here gives numerical results which are clearly in good agreement with the ones extracted from simulation in the previous section $\left(I_{\mathrm{NP}}^{g s}: I_{\mathrm{NP}}^{e s}=1: 0.16\right)$, given the approximation made. This strengthens our attribution of excited states to originate from the quasifree exciton orbiting around the neutral acceptor. In other words, the observed excited states can be associated with the second orbital of an exciton interacting with a sort of central potential around the impurity.

We end this part by trying to directly, i.e., without empirical broadening functions, describe the spectral lineshapes of phonon replicas. They are obtained after integration of the squared matrix element $|F(\mathbf{K})|^{2}$ along the transverse wave vector $\left(K_{x}\right.$ and $\left.K_{y}\right)$ directions, where "transverse" means perpendicular to $K_{z}$. The integration is calculated as $\int_{k_{\min }}^{\infty} 2 \pi\left|F\left(k+k_{0 z}\right)\right|^{2} k d k$, which depends on the lower limit $k_{\min }=\left|\left(E^{ \pm}-E_{N P} \mp E_{v}\right) / g_{\nu}\right|$ and thus on $E^{ \pm}$given by Eq. (2). Rewriting $E^{ \pm}$by $E$ and $E_{\mathrm{NP}} \pm E_{v}$ by $E_{0}$, the phonon replica lineshape is finally expressed by analytic forms

$$
\begin{gathered}
\mathcal{L}_{v}^{1 s}(E)=\frac{\pi}{3\left|g_{v}\right|} \frac{1}{\left(1+z^{2}\right)^{3}}, \\
\mathcal{L}_{v}^{2 s}(E)=\frac{16 \pi}{15\left|g_{v}\right|} \frac{1-10 z^{2}+40 z^{4}}{\left(1+4 z^{2}\right)^{5}}, \\
\mathcal{L}_{v}^{2 p}(E)=\frac{8 \pi}{15\left|g_{v}\right|} \frac{1+20 z^{2}}{\left(1+4 z^{2}\right)^{5}},
\end{gathered}
$$

where $z=\left|\left(E-E_{0}\right) / g_{v}\right| a_{B}$. These spectral shapes of phonon replicas are plotted in Fig. 4(c).

It is worthwhile mentioning that the phonon replicas of the $2 s$ and $2 p$ states are found with an integrated intensity identical to that of the $1 s$ state [as can be seen by integrating Eqs. (7) to (9) or graphically on Fig. 4(c)]. This contrasts with the NP line intensities, which are strongly attenuated for the excited $(2 s)$ states compared to the ground $(1 s)$ states. The fact that $I^{1 s}=I^{2 p}=I^{2 s}$ for phonon-assisted transition accounts well for the intense lines associated to the excited states in the absorption spectrum. The description of the phonon replicas with hydrogen functions then appears quite satisfactory since it accounts well for the order of magnitude of intensities observed in Fig. 2(b) and quantitatively extracted from fits in Sec. III-B: $I_{v}^{g s}: I_{v}^{e s}=I_{\mathrm{NP}}^{g s} A_{v}^{g s}: I_{\mathrm{NP}}^{e s} A_{v}^{e s}=1: 2$. Contrary to NP lines, both $2 s$ and $2 p$ excited states can contribute to phonon-assisted transitions, although fitting using Eqs. (8) and (9) was made difficult by the unknown $2 p$ state energy.
The inset in Fig. 4(c) compares a Gaussian (the dashed line), a Laplacian (the dashed-dotted line), and Eq. (7) (the full line), respectively, convoluted by Gaussian NP lines. The tails of Eq. (7) tend towards the Laplacian broadened line, which finally explains why this empirical function gives reasonable results.

\section{CONCLUSION}

We presented a detailed analysis of phonon-assisted absorption and luminescence spectra recorded for bound excitons in boron-doped diamond at low temperature. The luminescence spectra first have been fitted with a limited number of parameters, which well account for the lineshapes of the phonon replicas. The replica lineshapes are described as NP lines broadened by the empirical Laplacian function. Based on the fundamental mirror symmetry with the luminescence process, we could reveal the significant contribution of excited states of bound excitons in the absorption spectrum.

The excited states have turned out to be essential for understanding the absorption features and quantitatively describing the spectra. Using the intrinsic dispersion of phonons, we could further extract the momentum spread $\Delta k=2 \times$ $10^{9} \mathrm{~m}^{-1}$ of the bound-exciton wave function, which is three times higher than in silicon.

The quasifree exciton model in the weak phonon coupling regime has been developed up to a quantitative level. It provides evidence that the excited states of the bound exciton are well described by an exciton orbiting around the neutral acceptor rather than an internal excited state of the exciton. It further supports the attribution of the 5.40-eV absorption to excited states with a $2 s$ character. The proposed model also accounts well for the absorption intensity of excitedstate NP lines compared to that of the ground states. Finally, the lineshapes of phonon replicas extracted from this model were reported with analytic expressions. They a posteriori justify the use of Laplacian functions for simulating the bound exciton optical spectra.

More generally, the unique diamond properties (i.e., the large group velocities of phonons and strong localization of the acceptor states) have been decisive to confirm the importance of mirror symmetry and hydrogen wave functions for understanding and simulating correctly the optical spectra of excitons bound at impurity centers in indirect semiconductors. Our study on diamond provides a significant improvement in the description of such optical spectra, which had remained mainly qualitative since the 1970s [24]. We believe that our study can be extended to a large class of impurity-bound excitons in semiconductors.

\section{ACKNOWLEDGMENTS}

This work was partially supported by a Grant-in-Aid for Scientific Research (B) (Grant No. 17H02910) from JSPS, Japan, a Grant-in-Aid for Challenging Research (Exploratory) (Grant No. 19K21849) from JSPS, Japan, and PICS (Grant No. 200835) from Centre National de la Recherche Scientifique, France. 
(a)

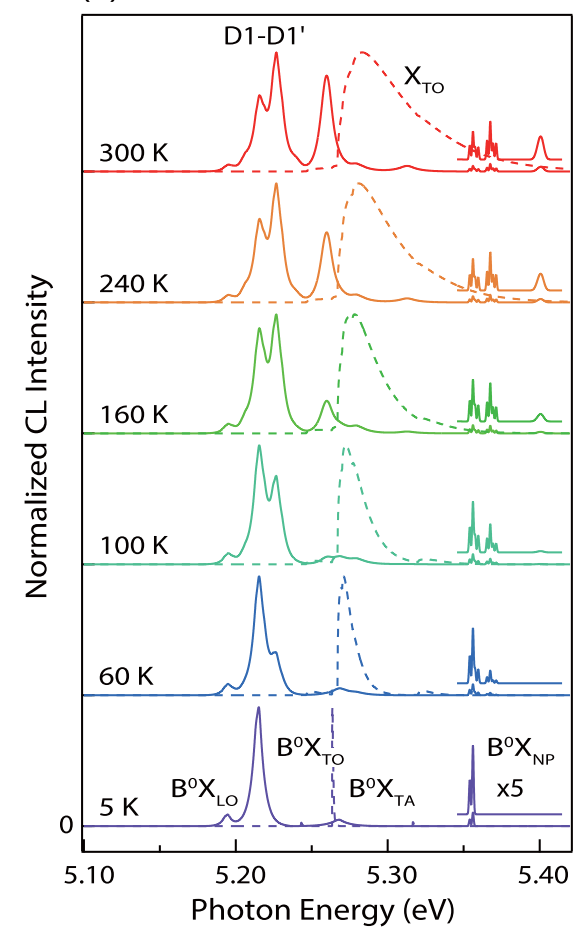

(b)

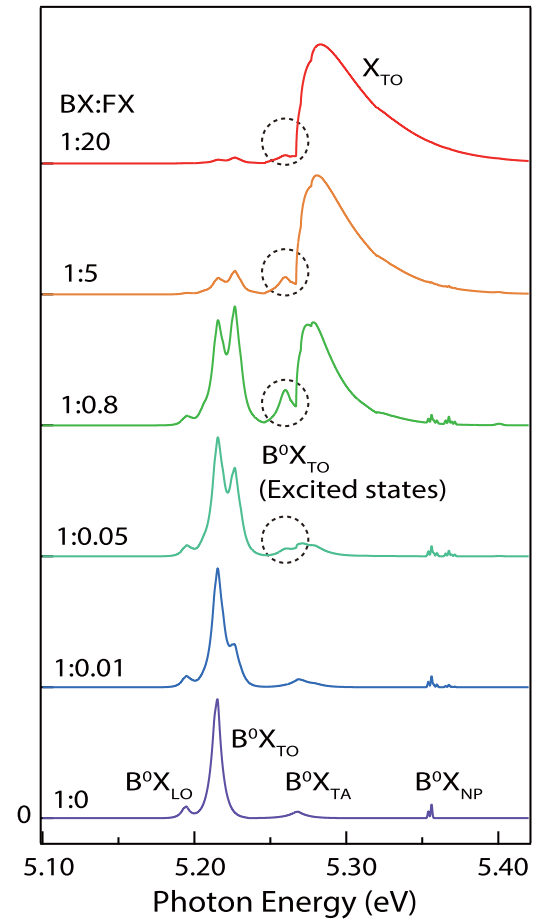

(c)



FIG. 5. Comparison of (a,b) calculated and (c) measured luminescence spectra of boron-doped diamond at various temperatures. In (a), the solid lines represent no-phonon lines and phonon replicas of the bound excitons whereas the dashed lines those of free excitons. In (b), the bound (BX) and free (FX) exciton contributions are summed with the weights indicated on each spectrum. The dotted circles in (b) and (c) evidence the contribution of excited states of boron-bound excitons observed with TO phonon replica.

\section{APPENDIX: TEMPERATURE-DEPENDENT LUMINESCENCE}

In Fig. 3(b), the shoulder indicated by an arrow is attributed to the TO phonon replica of the excited states. This is confirmed in Fig. 5, where we compare simulated and measured luminescence spectra at various temperatures.

In the simulation, the NP lines of the ground state were approximated by the sum of $2 \times 4$ Gaussians of equal widths but different amplitudes, as in Refs. [16,17]. The factor $2 \times$ comes from the final state splitting. To reproduce the temperature-dependent NP lines, we included the thermal activation of the fine structure of the ground state as well as of the excited states. The phonon replica lines were calculated by convoluting the NP lines with Laplacian broadening. The thermal activation of the $2 \times 4$ ground state also affects the TO-phonon replica, leading to the so-called $D_{1}-D_{1}^{\prime}$ doublet in the initial notations of Dean et al. [27].
In addition to the components originating from the bound excitons, the TO-phonon replica of the free excitons $X_{\mathrm{TO}}$ is overlaid by dashed lines in Fig. 5(a). Opposed to the almost temperature-independent linewidth of bound exciton lines, the phonon replicas of free (i.e., delocalized) excitons broaden with temperature reflecting the thermal population into high momentum states [18]. Figure 5(b) shows sums of the bound and free exciton components, with the weights between the two components indicated on each curve. These are in good agreement with the measured cathodoluminescence spectra in Fig. 5(c). This emphasizes that the TO phonon replica of the excited states should be included in the simulation to account for the peak at $5.26 \mathrm{eV}$, marked by dotted circles. More generally, the correct simulation of the bound-exciton luminescence spectra on a large range of temperature confirms the relevance of the quantitative analysis proposed in this work.
[1] D. F. Nelson and M. D. Sturge, Relation between absorption and emission in the region of the R lines of Ruby, Phys. Rev. 137, A1117 (1965).

[2] W. Gebhardt and H. Kühnert, Temperature dependence of Fcenter absorption and emission, Phys. Lett. 11, 15 (1964).

[3] I. Pelant and J. Valenta, Luminescence Spectroscopy of Semiconductors (Oxford University Press, New York, 2012).

[4] J. R. Haynes, Experimental Proof of the Existence of a New Electronic Complex in Silicon, Phys. Rev. Lett. 4, 361 (1960).
[5] M. L. W. Thewalt, D. Karaiskaj, T. Ruf, and M. Cardona, Proceedings of Int. Conf. Physics of Semiconductors (Edinburgh, 2002), (IOP, Bristol, England, 2002), p. 101.

[6] M. L. W. Thewalt, G. Kirczenow, R. R. Parsons, and R. Barrie, Phonon broadening of bound exciton luminescence in silicon, Can. J. Phys. 54, 1728 (1976).

[7] P. J. Dean, W. F. Flood, and G. Kaminsky, Absorption due to bound excitons in silicon, Phys. Rev. 163, 721 (1967). 
[8] K. R. Elliott, G. C. Osbourn, D. L. Smith, and T. C. McGill, Bound-exciton absorption in Si:Al, Si:Ga, and Si:In, Phys. Rev. B 17, 1808 (1978).

[9] D. S. Pan, D. L. Smith, and T. C. McGill, Concentration broadening of bound-excitons spectral lines, Phys. Rev. B 21, 3581 (1980).

[10] E. C. Lightowlers and Z. E. Ciechanowska, Acceptor bound exciton absorption and luminescence in germanium, J. Phys. C: Solid State Phys. 14, L719 (1981).

[11] A. T. Vink and R. C. Peters, Absorption and luminescence due to excitons bound to neutral acceptors in GaP, J. Lumin. 3, 209 (1970).

[12] K. Thonke, The boron acceptor in diamond, Semicond. Sci. Technol. 18, S20 (2003).

[13] Y. Kubo, S. Temgoua, R. Issaoui, J. Barjon, and N. Naka, Radiative lifetime of boron-bound excitons in diamond, Appl. Phys. Lett. 114, 132104 (2019).

[14] R. Issaoui, J. Achard, A. Tallaire, F. Silva, A. Gicquel, R. Bisaro, B. Servet, G. Garry, and J. Barjon, Evaluation of freestanding boron-doped diamond grown by chemical vapour deposition as substrates for vertical power electronic devices, Appl. Phys. Lett. 100, 122109 (2012).

[15] H. Kim, R. Vogelgesang, A. K. Ramdas, S. Rodriguez, M. Grimsditch, and T. R. Anthony, Electronic Raman and Infrared Spectra of Isotopically Controlled "Blue" Diamonds, Phys. Rev. Lett. 79, 1706 (1997).

[16] R. Sauer, H. Sternschulte, S. Wahl, K. Thonke, and T. R. Anthony, Revised Fine Splitting of Excitons in Diamond, Phys. Rev. Lett. 84, 4172 (2000).

[17] S. J. Sharp, A. T. Collins, G. Davies, and G. S. Joyce, Higher resolution studies of shallow bound exciton luminescence in diamond, J. Phys. Condens. Matter 9, L451 (1997).
[18] Y. Hazama, N. Naka, and H. Stolz, Mass-anisotropy splitting of indirect excitons in diamond, Phys. Rev. B 90, 045209 (2014).

[19] We note that the shoulder at the TO replica of the ground states should not be attributed to the LA phonon replica. If $W_{\mathrm{LA}} / W_{\mathrm{TO}}=2.5$ is assumed as we will see in Sec. IV, the coupling strength of the LA phonon replica $A_{\mathrm{LA}} / A_{\mathrm{TO}}$ is less than 0.04 to reproduce the luminescence, and its contribution can be neglected in the simulation of absorption and luminescence spectra.

[20] J. L. Warren, J. L. Yarnell, G. Dolling, and R. A. Cowley, Lattice dynamics of diamond, Phys. Rev. 158, 805 (1967).

[21] C. H. Henry and K. Nassau, Lifetimes of bound excitons in CdS, Phys. Rev. B 1, 1628 (1970).

[22] P. J. Dean, D. C. Herbert, C. J. Werkhoven, B. J. Fitzpatrick, and R. N. Bhargava, Donor bound-exciton excited states in zinc selenide, Phys. Rev. B 23, 4888 (1981).

[23] E. I. Rashba and G. E. Gurgenishvili, Edge absorption theory in semiconductors, Fizka Tverdogo Tela 4, 1029 (1962) [Soviet Phys. Solid State 4, 759 (1962)].

[24] P. J. Dean and D. C. Herbert, The localization of the lowest conduction band minima in gallium phosphide from bound exciton luminescence, J. Lumin. 14, 55 (1976).

[25] R. Szmytkowski, Alternative approach to the solution of the momentum-space Schrödinger equation for bound states of the N-dimensional Coulomb problem, Ann. Phys. (Berlin) 524, 345 (2012).

[26] Compared to the expression in Ref. [17], the normalization factor can differ by $\sqrt{4 \pi}$ depending on normalization of the spherical harmonics for the angular part.

[27] P. J. Dean, E. C. Lightowlers, and D. R. Wight, Intrinsic and extrinsic recombination radiation from natural and synthetic aluminum-doped diamond, Phys. Rev. 140, A352 (1965). 